\title{
Paradoxical Hyperadiponectinemia is Associated With the Metabolically Healthy Obese (MHO) Phenotype in African Americans
}

\author{
Ayo P. Doumateya, b, Amy R. Bentley, Jie Zhou a, Hanxia Huang, \\ Adebowale Adeyemo ${ }^{a}$, Charles N. Rotimi ${ }^{\mathrm{a}}$
}

\begin{abstract}
Background: It has been suggested that adiponectin may offer protection against the adverse health effects of obesity. In this study, we determined the prevalence of paradoxically high adiponectin or paradoxical hyperadiponectinemia (PHA) among obese African Americans and investigated its relationship with the metabolically healthy obese (MHO) phenotype.
\end{abstract}

Methods: Total adiponectin and metabolic markers including fasting glucose, insulin, serum lipids and obesity measures were determined in 822 unrelated participants from the Howard University Family Study (HUFS). Logistic regression models were used to evaluate the association between MHO phenotype and PHA while adjusting for relevant covariates.

Results: Overall, men had significantly lower adiponectin levels than women. However, adiponectin level was associated with obesity measures, glucose, insulin and insulin resistance index in both men and women. Equal proportion of the obese male and female subjects $(19.2 \%$; 66/343) had PHA; these obese individuals with PHA had a healthier metabolic profile including higher HDL-cholesterol, lower insulin levels and smaller waist circumference and insulin levels compared to those without PHA. Also, 28\% (96/343) of the study participants met the criteria of MHO phenotype. Inter-

Manuscript accepted for publication April 13, 2012

${ }^{\mathrm{a}}$ Center for Research on Genomics and Global Health, National Human Genome Research Institute, National Institutes of Health, Bethesda, MD, USA

${ }^{\mathrm{b}}$ Corresponding author: Ayo P. Doumatey, 12 South Drive, Building 12 A, Bethesda, MD 20892, USA. Email: doumateya@mail.nih.gov

doi: $10.4021 /$ jem $95 \mathrm{w}$ estingly, 42\% (28/66) of the obese individuals with PHA also had the MHO phenotype. Finally, the MHO phenotype was associated with PHA in both men and women.

Conclusions: These findings confirm the presence of MHO in African Americans and demonstrate the association of PHA with the MHO phenotype. In all, our findings along with other published results provide evidence for a more systematic investigation of the mechanisms underlying the protective function of adiponectin and its potential therapeutic applications in human metabolic disorders.

Keywords: Obesity; Paradoxical hyperadiponectinemia (PHA); Metabolically healthy obese phenotype (MHO)

\section{Introduction}

Adiponectin, one of the major active molecules produced by white adipose tissue (WAT), has been shown to be important in glucose homeostasis and lipid metabolism [1-3]. Unlike leptin and other adipokines, circulating levels of adiponectin are inversely correlated with obesity [4]. It is also the only adipokine that exhibits both anti-inflammatory and antiatherogenic properties [3, 5]. Adiponectin exerts its anti-inflammatory function by downregulating IL-6 production and by inducing the anti-inflammatory cytokine, IL-10 [6]. It has also been suggested that adiponectin may offer protection against the adverse health effects of obesity and obesity-related disorders [7, 8]. In adults, decreased circulating adiponectin has been associated with insulin resistance (IR), low HDL-cholesterol, type 2 diabetes (T2D) and cardiovascular diseases (CVD) [9, 10]. Furthermore, plasma adiponectin is inversely associated with serum triglycerides (TG) [11].

A number of studies in different populations have investigated the relationship between adiponectin, obesity markers, circulating lipids, IR, T2D and CVD $[9,12,13]$. Until recently, only few epidemiologic studies were available in African Americans (AA), and these were characterized by small sample sizes (ranging from 69 to 522 individuals) and the inclusion of limited numbers of clinical and biochemical measurements [10, 14-17]. In addition, most of 
Table 1. Anthropometric and Metabolic Characteristics of the 822 African American Participants Included in this Study

\begin{tabular}{|c|c|c|c|}
\hline Variable & $\operatorname{Men}(n=364)$ & Women $(n=458)$ & P-value \\
\hline Age (years) & $43.9 \pm 10.9$ & $42.8 \pm 10.7$ & 0.13 \\
\hline BMI $\left(\mathrm{kg} / \mathrm{m}^{2}\right)$ & $27.9 \pm 6.8$ & $31.3 \pm 8.8$ & $6.5 \times 10^{-10}$ \\
\hline PFM & $27.9 \pm 9.2$ & $41.1 \pm 9.3$ & $8.7 \times 10^{-73}$ \\
\hline $\mathrm{WC}(\mathrm{cm})$ & $93.3 \pm 15.9$ & $94.35 \pm 16.87$ & 0.35 \\
\hline WHR & $0.89 \pm 0.07$ & $0.84 \pm 0.07$ & $2.5 \times 10^{-26}$ \\
\hline Glucose (mg/dL) & $86.3 \pm 12.3$ & $83.7 \pm 10.2$ & 0.001 \\
\hline \multicolumn{4}{|l|}{ Insulin $(\mu \mathrm{U} / \mathrm{mL})$} \\
\hline Mean & $10.7 \pm 13.2$ & $12.3 \pm 18.2$ & 0.16 \\
\hline Geometric mean & 6.6 & 8.1 & \\
\hline Median (IQR) & $7.4(9.5)$ & $7.8(9.2)$ & \\
\hline \multicolumn{4}{|l|}{ HOMA-IR } \\
\hline Mean & $2.5 \pm 3.6$ & $2.7 \pm 4.7$ & 0.46 \\
\hline Geometric mean & 1.4 & 1.7 & \\
\hline Median (IQR) & $1.6(2.1)$ & $1.6(2.1)$ & \\
\hline HDL-C(mg/dL) & $52.9 \pm 19.2$ & $54.7 \pm 16.8$ & 0.15 \\
\hline LDL-C (mg/dL) & $111.6 \pm 40.8$ & $116.8 \pm 38.1$ & 0.06 \\
\hline \multicolumn{4}{|l|}{$\mathrm{TG}(\mathrm{mg} / \mathrm{dL})$} \\
\hline Mean & $111.3 \pm 69.6$ & $100.72 \pm 68.5$ & 0.03 \\
\hline Geometric mean & 97.7 & 87.1 & \\
\hline Median (IQR) & $95(62)$ & $83(54)$ & \\
\hline Total cholesterol (mg/dL) & $187.0 \pm 44.9$ & $192.4 \pm 42.6$ & 0.08 \\
\hline \multicolumn{4}{|l|}{ Adiponectin (ng/mL) } \\
\hline Mean & $7855.2 \pm 4704.0$ & $9186.3 \pm 5551.8$ & 0.0002 \\
\hline Geometric mean & 6760.8 & 7762.4 & \\
\hline Median (IQR) & $6527.9(5853)$ & $7734.7(6983)$ & \\
\hline Hypertension (\%) & 36.8 & 32.5 & \\
\hline $\mathrm{SBP}(\mathrm{mmHg})$ & $130.1 \pm 19.4$ & $82.0 \pm 13.6$ & 0.003 \\
\hline DBP $(\mathrm{mmHg})$ & $126.1 \pm 18.3$ & $79.8 \pm 11.8$ & 0.016 \\
\hline
\end{tabular}

Data is presented as mean \pm standard deviation; geometric means, median and interquartile ranges (IQR) are also presented when variables were not normally distributed. Student's t-tests were used to compare means between gender. 


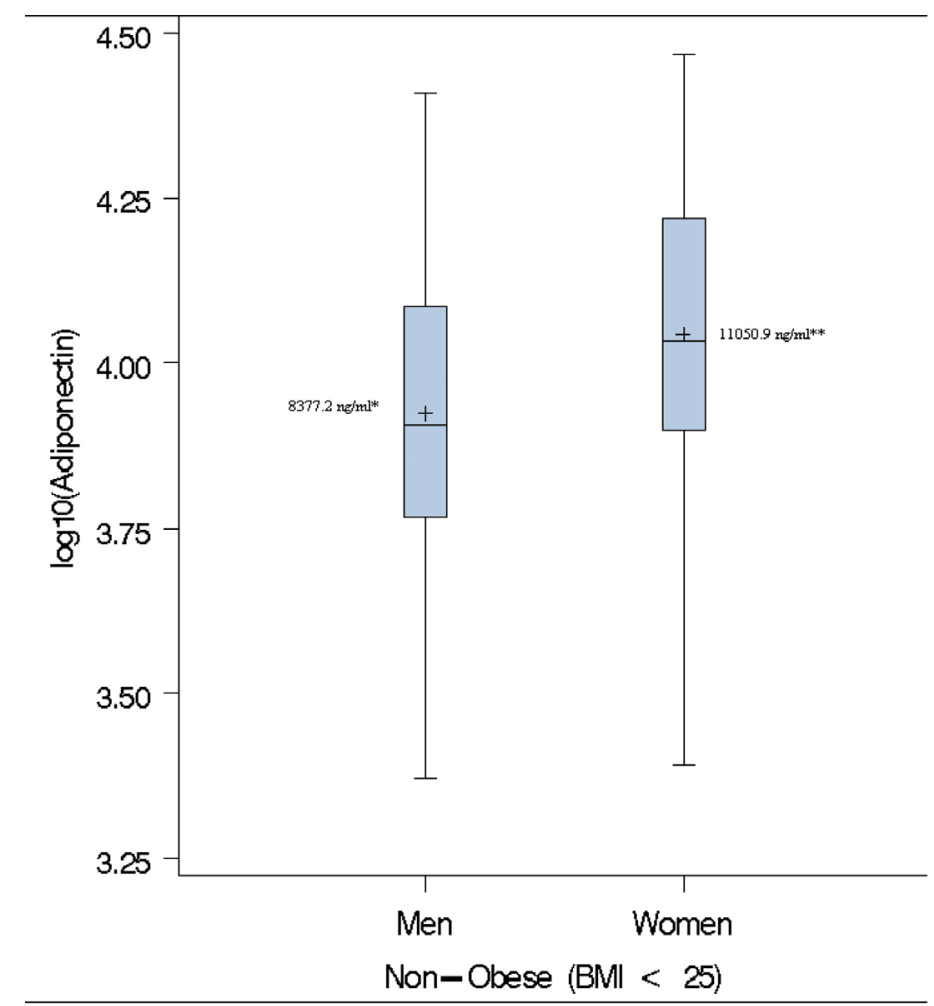

Figure 1. Box-plots of log adiponectin in lean individuals. (+) denotes the geometric mean; * Cut-off used to define paradoxical hyperadiponectinemia in Men; ${ }^{*}$ Cut-off used to define paradoxical hyperadiponectinemia in Women.

the studies published in AA were designed either to compare adiponectin and other adipokine levels or to investigate the variability of adiponectin levels across ethnic groups [14, 18]. It is only in the last couple of years that larger studies of AA have investigated the relationship between serum adiponectin, adiponectin gene variation and different phenotypes [19-23].

Serum adiponectin, a key modulator of metabolic pathways, is generally lower in AA than in Caucasians [18]. Paradoxically high adiponectin in the presence of a healthy metabolic profile is becoming an increasingly recognized concept $[24,25]$. Identification and characterization of individuals with higher-than-expected adiponectin levels (paradoxical hyperadiponectinemia, PHA) in metabolically healthy but obese (MHO) individuals is of great importance because it may provide insights into mechanisms by which some obese individuals are protected from a wide range of obesity-related complications. It may also enhance the ability to develop more focused obesity management strategies at the individual level. Currently, there is a paucity of published studies that have estimated the prevalence of PHA in obese adult AA or its relationships with the MHO phenotype.

The MHO phenotype was described by Karelis et al. as obese individuals with favorable metabolic profiles characterized by high insulin levels with normal lipid and hormonal profiles $[26,27]$. To our knowledge, the only such study in
AA was conducted by Morrison et al [25] in young AA girls and found that higher-than-expected serum adiponectin was associated with MHO. These investigators also observed that high adiponectin level protected the young AA obese girls against the development of metabolic syndrome later in life [28]. In addition, it is estimated that about $20-30 \%$ of obese adults [24] and young obese girls have paradoxically high adiponectin levels despite high BMI. High adiponectin levels have been associated with metabolically healthy phenotype which is characterized by high HDL-C, low insulin, TG and glucose $[25,28]$. In this study, we aimed to determine the prevalence of PHA among obese AA and its relationship with the MHO phenotype using a population-based sample of AA enrolled from the Washington DC metropolitan area.

\section{Methods and Procedures}

\section{Study subjects}

Subjects included in this study were participants in the Howard University Family Study (HUFS) which has been fully described elsewhere [29]. Briefly, the HUFS is a populationbased family study of AA in the Washington, D.C. metropolitan area The major objective of the HUFS was to enroll and examine a randomly ascertained sample of AA families 

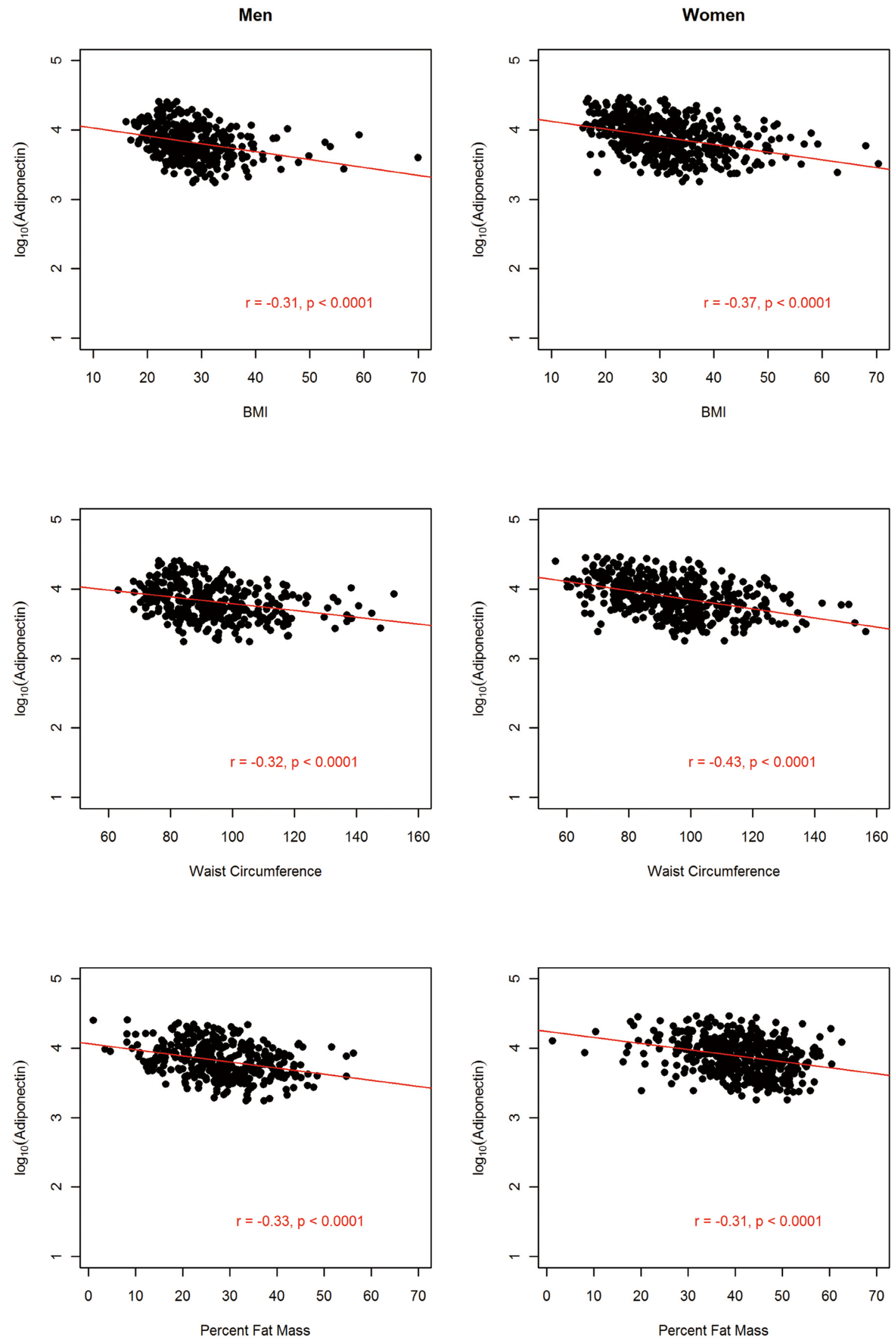

Figure 2. Association between circulating adiponectin levels and obesity markers by gender in 822 African Americans. 

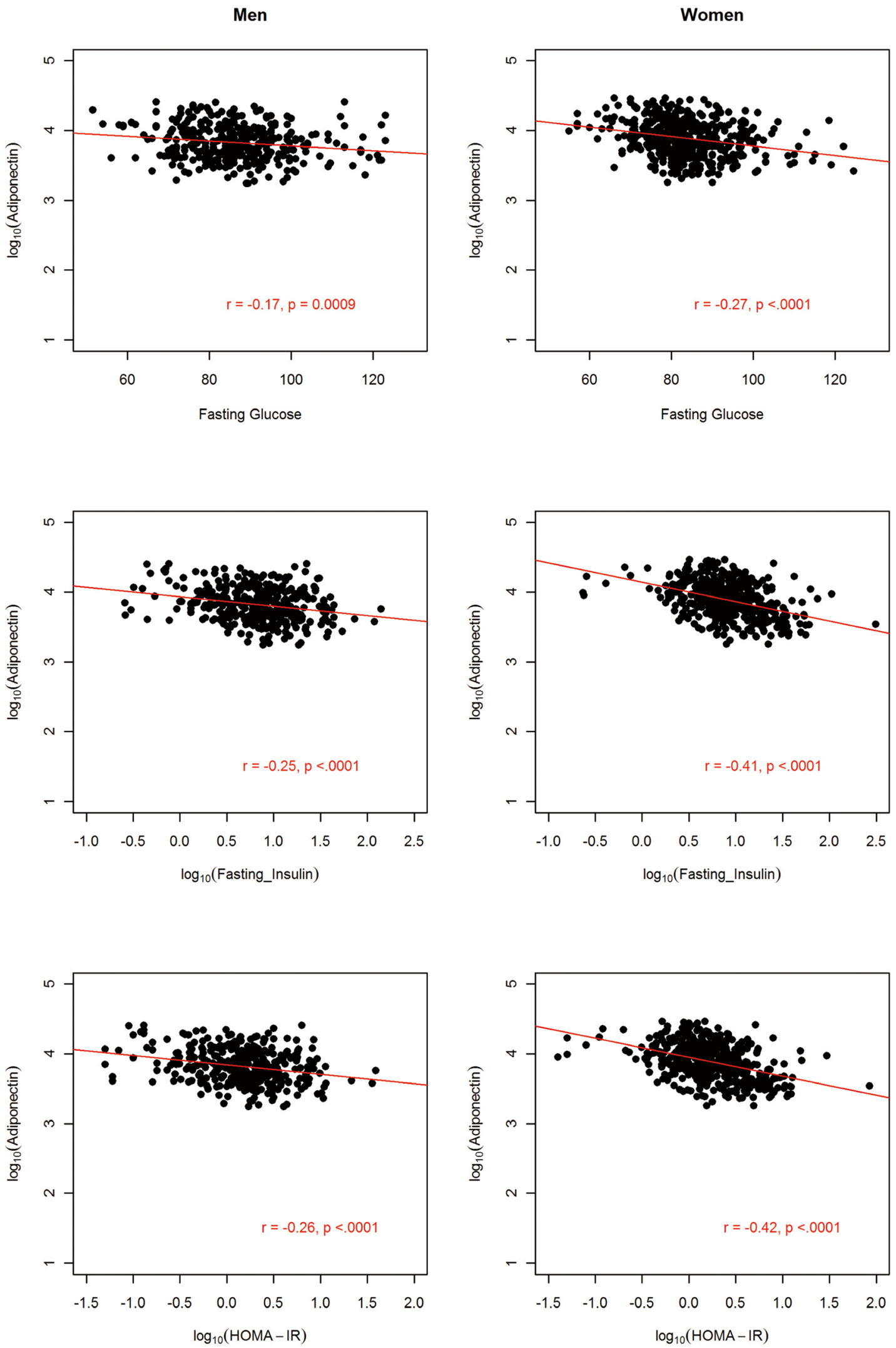

Figure 3. Association between circulating adiponectin levels and glycolytic indexes by gender in 822 African Americans. 

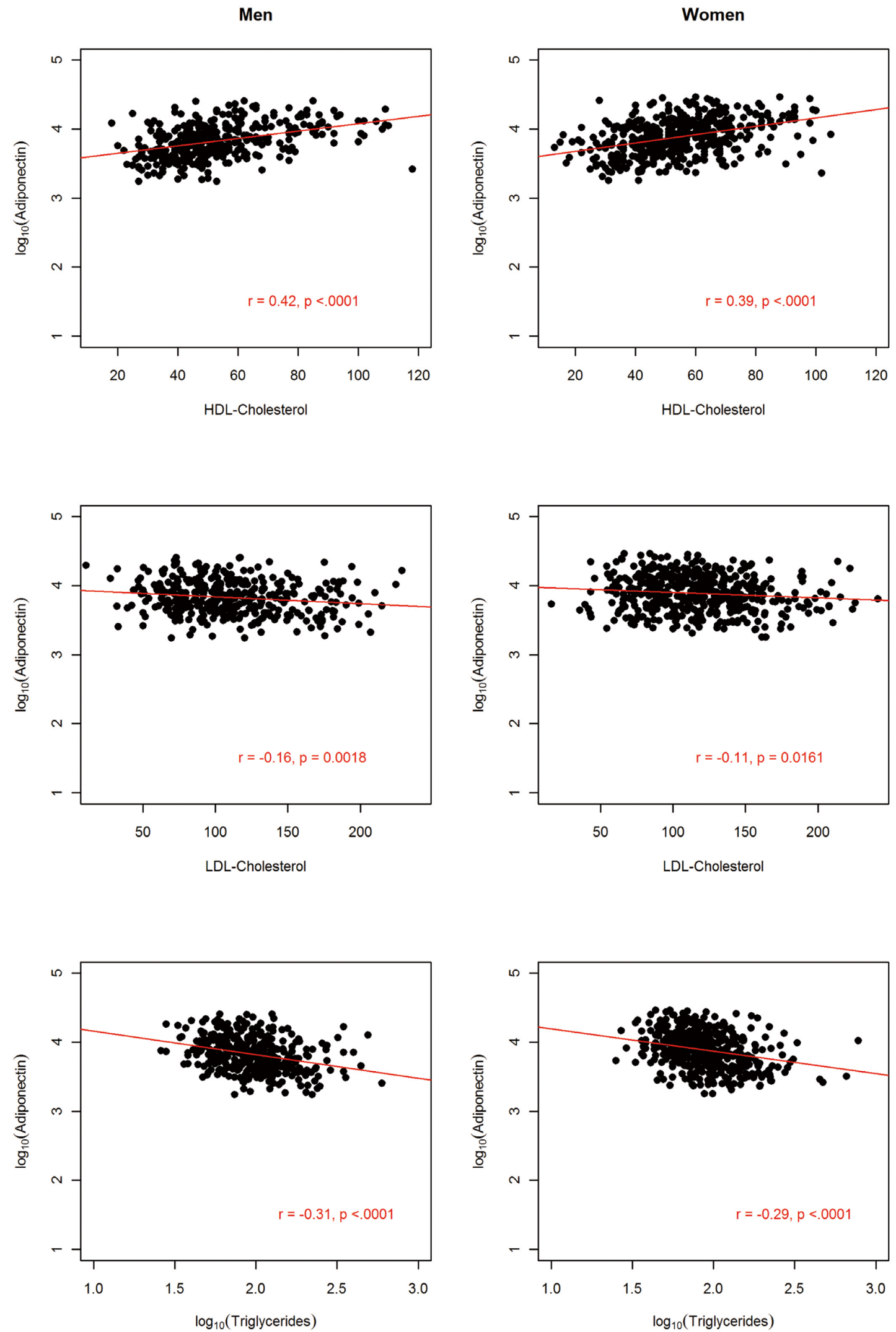

Figure 4. Association between circulating adiponectin levels and serum lipids by gender in 822 African Americans. 
Table 2. Clinical Characteristics of the Obese African American Subjects Included in this Study

\begin{tabular}{|c|c|c|c|}
\hline Variables & $\operatorname{Men}(n=110)$ & Females $(n=233)$ & P-value \\
\hline Age (years) & $42.9 \pm 10.5$ & $42.8 \pm 9.8$ & 0.95 \\
\hline BMI $\left(\mathrm{kg} / \mathrm{m}^{2}\right)$ & $35.7 \pm 6.5$ & $38.0 \pm 7.0$ & 0.004 \\
\hline PFM & $36.0 \pm 7.5$ & $46.6 \pm 6.4$ & 0.000 \\
\hline $\mathrm{WC}(\mathrm{cm})$ & $110.9 \pm 14.4$ & $106.0 \pm 13.4$ & 0.002 \\
\hline WHR & $0.94 \pm 0.06$ & $0.85 \pm 0.07$ & $<0.0001$ \\
\hline $\mathrm{SBP}(\mathrm{mmHg})$ & $131.8 \pm 17.4$ & $129.4 \pm 18.5$ & 0.26 \\
\hline DBP (mmHg) & $83.4 \pm 11.0$ & $81.0 \pm 11.6$ & 0.08 \\
\hline Glucose (mg/dL) & $90.9 \pm 12.3$ & $86.0 \pm 9.8$ & $<0.0001$ \\
\hline Insulin $(\mu \mathrm{U} / \mathrm{mL})$ & $16.8 \pm 18.9(12.6)$ & $16.4 \pm 23.7(11.5)$ & 0.87 \\
\hline HOMA-IR & $4.01 \pm 5.4(2.7)$ & $3.7 \pm 6.3(2.4)$ & 0.61 \\
\hline HDL-C(mg/dL) & $43.8 \pm 11.3$ & $51 \pm 15.3$ & $<0.0001$ \\
\hline LDL-C (mg/dL) & $126.7 \pm 44.4$ & $120.3 \pm 39.5$ & 0.18 \\
\hline $\mathrm{TG}(\mathrm{mg} / \mathrm{dL})$ & $128.2 \pm 77.9(109.6)$ & $105.1 \pm 63.8(93.3)$ & 0.007 \\
\hline Total cholesterol (mg/dL) & $196.1 \pm 47.7$ & $192.3 \pm 43.8$ & 0.46 \\
\hline Adiponectin (ng/mL) & $6023.8 \pm 3227.1(5248.1)$ & $7541.2 \pm 4759.0(6309.6)$ & 0.001 \\
\hline
\end{tabular}

Data is presented as mean \pm standard deviation; values in parenthesis ( ) are geometric means when variables were not normally distributed; Student's t-test was used to assess difference between men and women.

along with a set of unrelated individuals for study of the genetic and environmental bases of common complex traits including hypertension, obesity, diabetes, and associated phenotypes. In order to maximize the utility of this cohort for the study of multiple common traits, families and individuals were not ascertained based on any phenotype. All participants were recruited after an overnight fast of at least 8 hours prior to the blood draw and all collected samples were stored in a $-80{ }^{\circ} \mathrm{C}$ freezer pending measurement of biochemical parameters. Study protocol was approved by the Institutional Review Board (IRB) at Howard University and all study participants provided written informed consent. The present study of PHA included only unrelated and nondiabetic participants from the overall HUFS.

\section{Anthropometric measurements}

Weight was measured in light clothing with an electronic scale to the nearest $0.1 \mathrm{~kg}$, height was measured with a stadiometer to the nearest $0.1 \mathrm{~cm}$, and BMI was calculated as weight in $\mathrm{kg}$ divided by height in meters square $\left(\mathrm{kg} / \mathrm{m}^{2}\right)$. Waist circumference (WC) was measured to the nearest 0.1 
$\mathrm{cm}$ at the narrowest part of the torso. Body composition was estimated using bioelectric impedance (BIA) analysis with validated population specific equation as previously described [30] and percentage fat mass (PFM) was obtained using the following formula: (Fat mass/weight) x 100 .

\section{Measurement of metabolic markers and adiponectin}

HDL-cholesterol (HDL-C), LDL-cholesterol (LDL-C), triglycerides (TG) and glucose were determined enzymatically with COBAS Integra Plus Analyzer ${ }^{\circledR}$ (Roche Diagnostics, Indianapolis, IN, USA). Insulin was measured using an immunoassay analyzer, Elecsys 1010 (Roche Diagnostics, Indianapolis, IN). IR was assessed by the HOMA-IR method. In HOMA-IR, values are calculated from the fasting concentrations of insulin and glucose using the following formula: (Fasting serum insulin $(\mathrm{mU} / \mathrm{L}) \mathrm{x}$ Fasting plasma glucose $(\mathrm{mmol} / \mathrm{L})) / 22.5$ as previously reported [31]. Adiponectin was measured using an enzyme-linked immunoabsorbent assay, ELISA (Quantikine ${ }^{\circledR}$ human adiponectin immunoassay, $\mathrm{R}$ and D Systems, Minneapolis, Minnesota, USA) following the manufacturer's recommendations.

\section{Definitions}

As previously described [24, 25, 28], paradoxical hyperadiponectinemia (PHA) in obese subjects was defined as adiponectin levels higher than the median of adiponectin in non-obese (BMI $<25 \mathrm{~kg} / \mathrm{m}^{2}$ ) subjects. In the present study, the adiponectin levels followed a log-normal distribution. Therefore, we opted to use the geometric mean instead of the median as the measure of central tendency. The geometric mean adiponectin level in non-obese subjects used as threshold for PHA in this study were $8,377.2 \mathrm{ng} / \mathrm{mL}$ for men and $11,050.9 \mathrm{ng} / \mathrm{mL}$ for women, respectively (Fig. 1). The MHO phenotype was defined as the concomitant presence of BMI $\geq 30 \mathrm{~kg} / \mathrm{m}^{2}$, fasting plasma glucose $\leq 126 \mathrm{mg} / \mathrm{dL}$, systolic blood pressure $(\mathrm{SBP}) \leq 130$ or diastolic blood pressure $(\mathrm{DBP}) \leq 85, \mathrm{HDL}-\mathrm{C} \geq 40 \mathrm{mg} / \mathrm{dL}$ for men and HDL-C $\geq 50$ $\mathrm{mg} / \mathrm{dL}$ for women [24].

\section{Statistical analysis}

All statistical analysis were performed using SAS software version 9.1 (SAS Institute, Cary, North Carolina, US). Graphics were generated using the R package (www.R-project.org). All variables that deviated from normal distribution were logarithmically-transformed to normality before conducting any statistical analysis. Continuous variables are expressed as mean \pm standard deviation (SD) or as geometric means, medians and interquartile ranges. Student's t-test was used to compare study variables between gender. Pearson correlation coefficients (r) and scatter plots were used to assess the relationship between adiponectin, obesity measures
(BMI, WHR, and PFM) and metabolic markers ( HDL-C, LDL-C, triglycerides (TG), glucose and HOMA-IR) separately for men and women. Logistic regression models were used to determine the relationships between MHO and PHA with models adjusted for age and additional covariates (hypertension, WC, and insulin) which were added sequentially to evaluate how each covariates modified observed associations.

\section{Results}

Clinical characterization and relationship between adiponectin and metabolic markers in the entire cohort

The sample comprised 822 AA participants (364 men and 458 women). The anthropometric and metabolic characteristics of the study participants, stratified by gender, are summarized in Table 1. On average, men had significantly higher fasting glucose, triglycerides, and WHR and the prevalence of hypertension was about $4 \%$ higher in men than in women. In contrast, women were more obese, as exemplified by higher BMI (31.3 vs. $\left.27.9 \mathrm{~kg} / \mathrm{m}^{2}\right)$ and higher PFM (41.1\% vs. $27.9 \%$ ). Despite this, women had higher adiponectin level compared to the males (mean difference $=1,001.6 \mathrm{ng} / \mathrm{mL}$; $\mathrm{P}=0.0005)$. Consistent with previous findings, adiponectin was negatively associated with obesity measures (BMI, WC and PFM), fasting glucose, insulin, HOMA-IR and triglycerides but positively associated with HDL-C in both men and women (Fig, 2, 3, 4).

\section{Prevalence of paradoxical hyperadiponectinemia (PHA) in obese subjects}

The prevalence of obesity in the study sample was $41.7 \%$ $(343 / 822)$ and was nearly two times higher in women than in men $(50.9 \%$ vs. $30.2 \%)$. The clinical characteristics of obese subjects are summarized in (Table 2). Mean BMI among obese subjects was $37.2 \pm 6.9 \mathrm{~kg} / \mathrm{m}^{2}$ and similar gender differences were observed among the obese subjects as were seen in the overall cohort (i.e. women were heavier, but had a healthier metabolic profile and higher adiponectin levels).

The gender-specific geometric means of adiponectin in the non-obese group i.e. BMI $<25 \mathrm{~kg} / \mathrm{m}^{2}(8,377.2 \mathrm{ng} / \mathrm{mL}$ for men and 11,050.9 ng/mL for women) were used to characterize subjects with PHA (Fig. 1). The overall prevalence of PHA among obese subjects was $19.2 \%$ (66/343; women $=19.3$ and men $=19.1 \%$, Table 3 ). On average, adiponectin level was 2.5 times higher in individuals with paradoxically high adiponectin. The inverse relationship between adiponectin and BMI was maintained in obese individuals with PHA (partial coefficient of correlation, $\mathrm{r}_{\text {age, sex }}=-0.31, \mathrm{P}=$ 0.01). Individuals with PHA had higher HDL-C and lower 


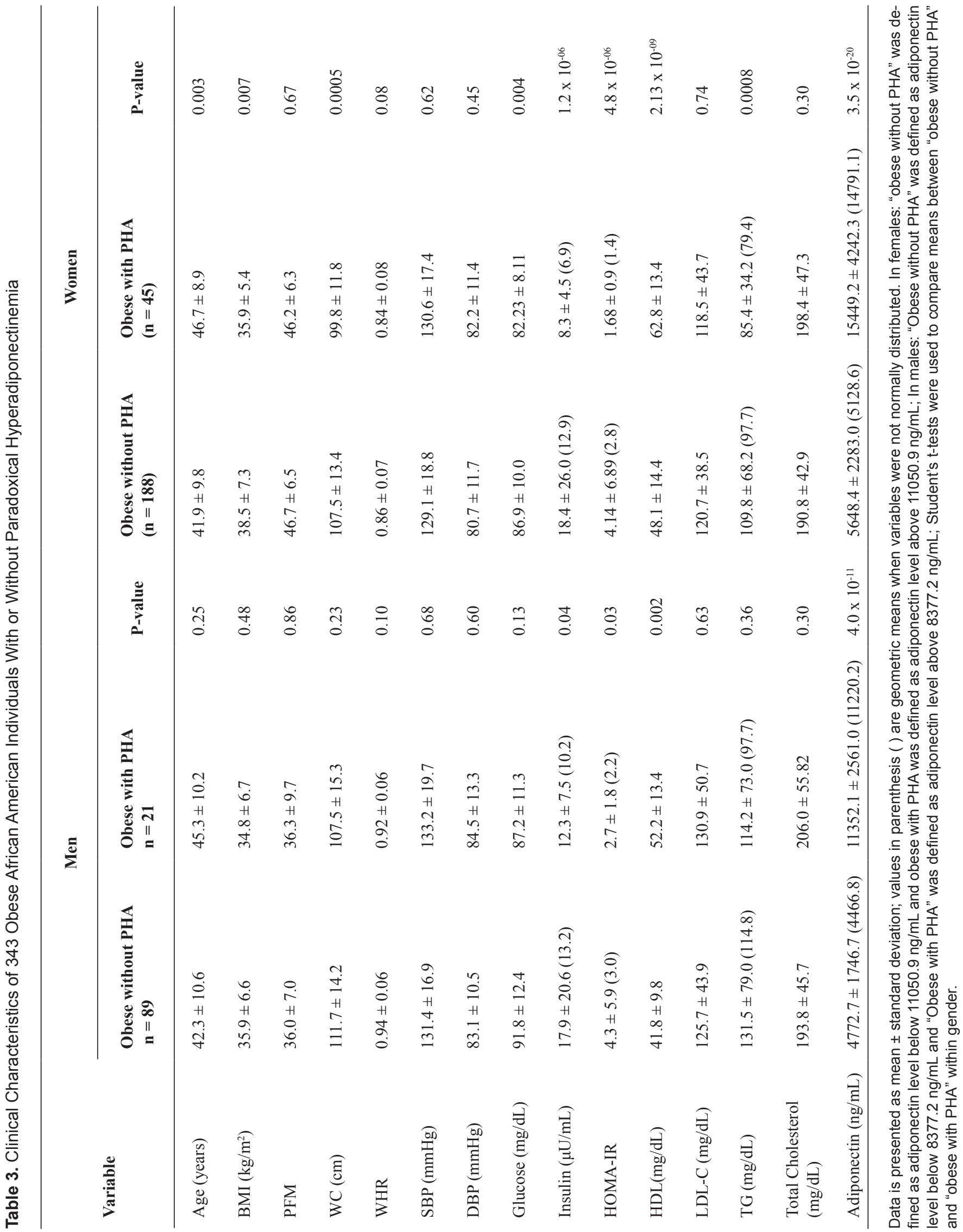




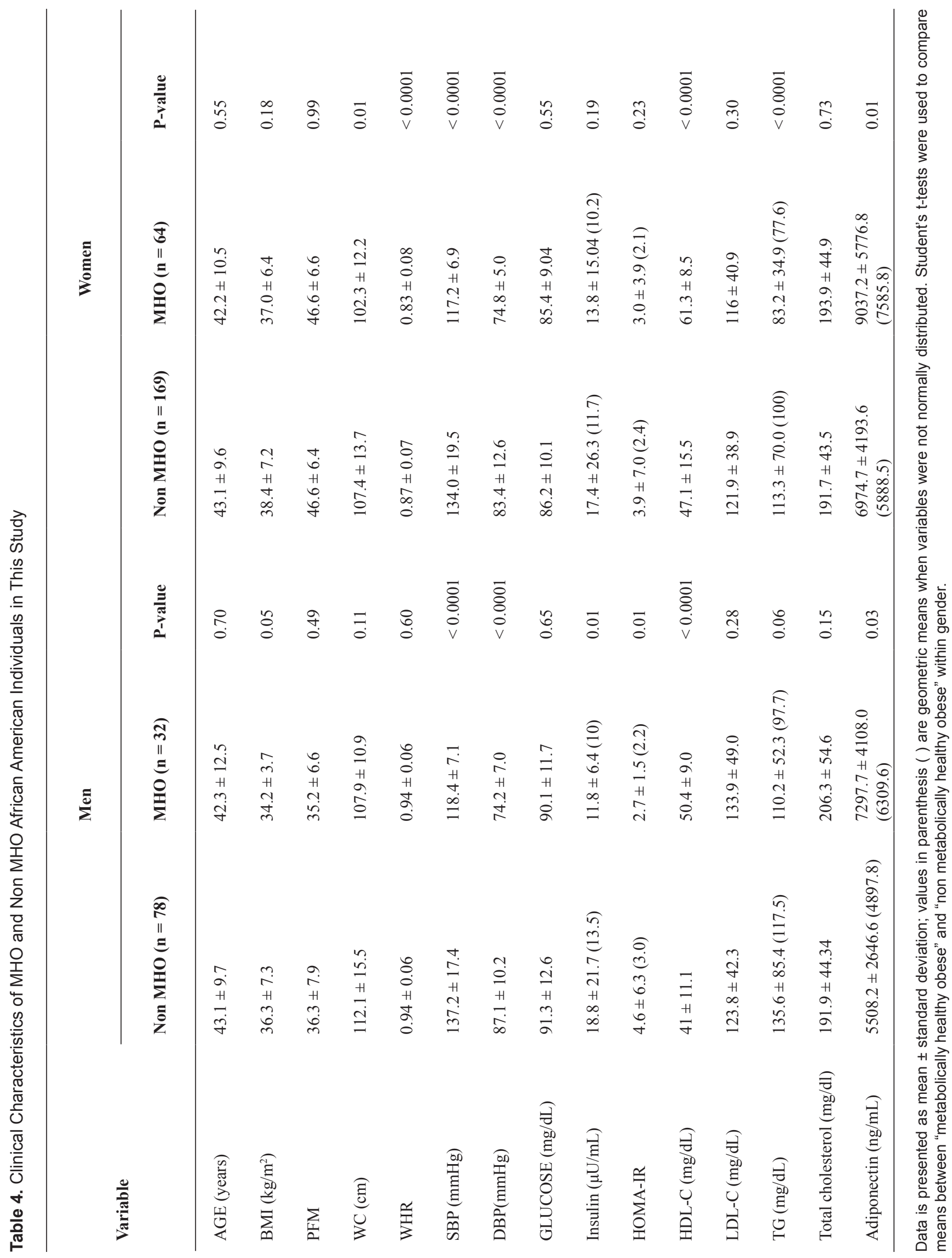


Table 5. Association Between MHO Phenotype and Adiponectin Levels Among African Americans in Logistic Regression Models With Adjustment for Relevant Covariates

Dependant variable: MHO $($ yes $=1 ;$ no $=0)$

\begin{tabular}{|c|c|c|c|c|}
\hline Gender & PHA: yes/no (covariates) & B \pm S.E. & OR $(95 \% \mathrm{CI})$ & P-value \\
\hline \multirow{5}{*}{ Men } & $\mathrm{PHA}^{*}$ & $1.01 \pm 0.50$ & $2.8(1.03-7.40)$ & $0.04 *$ \\
\hline & PHA $^{¥}$ ( age) & $1.10 \pm 0.51$ & $2.9(1.1-7.80)$ & $0.04 *$ \\
\hline & PHA $^{*}$ (age, HTN) & $1.30 \pm 0.56$ & $3.5(1.2-10.55)$ & $0.03 *$ \\
\hline & PHA ${ }^{\ddagger}$ (age, HTN, WC) & $1.20 \pm 0.57$ & $3.3(1.1-10.02)$ & $0.04 *$ \\
\hline & $\mathrm{PHA}^{\ddagger}$ (age, HTN, WC, INS) & $1.30 \pm 0.59$ & $3.6(1.1-11.5)$ & $0.03 *$ \\
\hline \multirow{5}{*}{ Women } & $\mathrm{PHA}^{*}$ & $0.72 \pm 0.35$ & $2.1(1.04-4.10)$ & $0.04 *$ \\
\hline & $\mathrm{PHA}^{¥}$ (age) & $0.80 \pm 0.4$ & $2.2(1.10-4.51)$ & $0.03 *$ \\
\hline & PHA $^{*}$ (age, HTN) & $0.90 \pm 0.39$ & $2.4(1.10-5.03)$ & $0.03 *$ \\
\hline & PHA $^{\ddagger}$ (age, HTN, WC) & $0.65 \pm 0.40$ & $1.9(0.90-4.20)$ & 0.10 \\
\hline & PHA ${ }^{*}$ (age, HTN, WC, INS) & $0.61 \pm 0.41$ & $1.8(0.80-4.12)$ & 0.14 \\
\hline
\end{tabular}

B: coefficient, S.E. standard error of B, OR: estimated odds ratio, Cl: confidence interval of OR; HTN: hypertension $(0=$ no hypertension, 1 = hypertension), WC = waist circumference, INS = log- insulin; $¥$ PHA status was coded 1, if adiponectin level is above $8377.2 \mathrm{ng} / \mathrm{mL}$ and $11050.9 \mathrm{ng} / \mathrm{mL}$ for men and women respectively; or 0 , if adiponectin level is below $8377.2 \mathrm{ng} / \mathrm{mL}$ and 11050.9 $\mathrm{ng} / \mathrm{mL}$ for men and women respectively. ${ }^{*}$ Denote significant $\mathrm{P}$-value $(\mathrm{P}<0.05)$

WC, TG, glucose, serum insulin and fasting glucose compared to those without PHA (Table 3).

\section{Association between MHO and paradoxical hyperadipo- nectinemia (PHA)}

The MHO phenotype was present in 96 obese individuals representing $28 \%$ of the obese subjects (men $=29.1 \%$ and women $=27.5 \%$; Table 4). Among men, MHO individuals had significantly lower insulin, were more insulin sensitive as measured by HOMA-IR and had significantly higher adiponectin than non MHO individuals. Among women, WHR, and TG were significantly lower and adiponectin higher in MHO individuals than non MHO but the difference in in- sulin and HOMA-IR did not reach significance among the two groups (Table 4). Twenty-eight of the sixty-six (42.4\%) individuals with PHA were also metabolically healthy obese.

Using logistic regression models, we found that MHO was significantly associated with PHA in both men $(\mathrm{OR}=$ $2.8,95 \%$ confidence interval $(1.03-7.40)$ ) and women (OR $=2.1,95 \% \mathrm{CI}(1.04-4.10)$; Table 5$)$. This association was stronger $(\mathrm{OR}=3.6,95 \%$ CI $(1.14-11.5))$ in men after adjusting for age, WC, insulin and hypertension. In women, the association remained significant after adjusting for age and hypertension; surprisingly however, the models that included either WC $(\mathrm{OR}=1.91,95 \% \mathrm{CI}(0.9-4.2))$ or insulin $(\mathrm{OR}=2.1,95 \% \mathrm{CI}(0.96-4.6) ; \mathrm{P}=0.06)$ did not reach significance in women. 


\section{Discussion}

The importance of adiponectin as a clinical biomarker is gaining increasing recognition. In this study, we sought to characterize PHA and to evaluate its association with the metabolically healthy obese (MHO) phenotype in a large well-characterized population-based sample of AA adults. Previous studies have found an association between PHA and MHO in other populations [24, 25]. This study is the first to investigate PHA in a large cohort of adult AA with a focus on identifying gender differences. We found that all obesity measures were negatively associated with adiponectin levels as previously reported [13, 15, 16, 32]. Women had higher circulating adiponectin levels than men despite having higher BMI and PFM also as previously observed in other studies [15, 32].

As previously shown in young AA girls $[25,28]$ and in Hispanics [24], we observed PHA in obese men and women. However, despite having differences in the degree of obesity, the prevalence of PHA was not significantly different between obese men and women in this study. This may be due to the differences in the distribution and localization of fat. Notably, although men and women in this study had almost the same mean waist circumference, women had significantly lower WHR consistent with the "pear shape" variety that is less associated with obesity-related complications including diabetes [33]. We speculate that the localization of fat in these obese women did not extensively impair their ability to efficiently produce adiponectin.

The prevalence of $19.2 \%$ of PHA in this study is lower than previously observed in adults [24, 25]. While this may be a unique characteristic of this cohort, it is consistent with the finding that, on average, adiponectin levels are lower in AA than other ethnic groups [18]. Therefore, our findings may simply be reflecting the overall lower adiponectin levels among AA.

A key finding in this study is that obese AA with PHA had a healthier metabolic profile. This implies that PHA should be considered an important characteristic of the metabolically healthy obese (MHO) phenotype. Furthermore, our analyses showed a strong association between PHA and the MHO phenotype in both men and women. In fact, individuals with PHA are two to three times more likely to be MHO than others. The differences between men and women on adjusting for covariates suggests that, unlike in men, the association between PHA and MHO in women is mainly driven by both visceral adiposity and insulin levels. This finding implies the involvement of more complex physiological interactions in women than in men.

The current criteria used to define the metabolic syndrome remain sub-optimal when used for individuals from populations of African descent [34]. Since the criteria often used to characterize MHO phenotype are closely related to those of metabolic syndrome, it is possible that current stud- ies, including the present one, may not be characterizing MHO optimally. The standardization of the criteria used to define MHO individuals need to be addressed in future studies, even though it is well-accepted that it remains hard to standardize the identification of MHO subjects [27]. In this regard, it is pertinent to note that it may be useful to include inflammatory markers (e.g. C-reactive protein, fibrinogen, white cell count (WBC)) since inflammation is a characteristic hallmark of obesity and obesity-related morbidities [35]. Assessing the predictive power of the criteria currently used to characterize MHO subjects will be key in clearly understanding the phenotype, especially at the genetic level. Although the MHO phenotype has been described [36], no studies have provided the molecular mechanisms linking high adiponectin levels with favorable metabolic profile. In this regard, more molecular studies including proteomics, metabolomics and genomics are needed to adequately identify this subset of obese individuals with the long term goal of understand the physiology involved in protecting these individuals from adverse conditions associated with obesity.

A limitation of this study is the fact that only total adiponectin (and not its multimeric forms) was measured. Studies that have measured isoforms of adiponectin have shown a more nuanced view of its associations with metabolic outcomes. For example, differences in low molecular weight (LMW) adiponectin explained the observed difference in adiponectin levels between AA and European Americans [37]. A second limitation is that the cross-sectional nature of this study limits any strong inference regarding causality. Recent studies have shown that a number of factors including physical activity, smoking, therapeutic agents and androgens that were not measured in this study affect the level of circulating adiponectin [38-40] and not controlling for such factors may affect observed associations. Lastly, even though the definition of PHA used in this study and previously published articles $[24,25]$ uses the lean individuals adiponectin mean as reference, it may not completely captured the variability and biological effect of adiponectin levels across obese individuals.

In summary, we demonstrated that adiponectin is associated with obesity and metabolic parameters (lipids, glucose and insulin resistance) in a large population-based cohort of AA. We showed that PHA is fairly common in obese AA and that it is associated with MHO phenotype. Notably, this is the first study to closely investigate the sexual dimorphic (male-females) effects on the relationship between PHA and obesity-related traits among AA, taking advantage of a large sample size, a wide age range (18 to 67 years), and focusing on within-population differences. Furthermore, we confirm, for the first time, the existence of the MHO phenotype in a population-based sample of AA. This study not only complements published data, it also allows generalization of the paradigm of PHA to non-European ancestry populations. Studying the relationship between high adiponectin and the 
phenomenon of $\mathrm{MHO}$ in AA is a significant topic with major public health importance that could lead to more targeted interventions directed at reducing the negative health impacts of obesity.

\section{Acknowledgement}

The study was supported by National Institutes of Health grants S06GM008016-320107 to CNR and S06GM008016-380111 to AA. Participants were enrolled at the Howard University General Clinical Research Center, which is supported by grant 2M01RR010284 from the National Center for Research Resources (NCRR), a component of National Institutes of Health (NIH). The contents of this publication are solely the responsibility of the authors and do not necessarily represent the official view of NCRR or NIH. This research was supported in part by the Intramural Research Program of the Center for Research on Genomics and Global Health. The Center for Research on Genomics and Global Health is supported by the National Human Genome Research Institute, the National Institute of Diabetes and Digestive and Kidney Diseases, the Center for Information Technology and the Office of the Director at the National Institutes of Health (Z01HG200362).

\section{Disclosure}

The authors declared no conflict of interest.

\section{Grant Support}

The study was supported by National Institutes of Health grants S06GM008016-320107 to CNR and S06GM008016-380111 to AA. Participants were enrolled at the Howard University General Clinical Research Center, which is supported by grant 2M01RR010284 from the National Center for Research Resources (NCRR), a component of National Institutes of Health (NIH). This research was supported in part by the Intramural Research Program of the Center for Research on Genomics and Global Health. The Center for Research on Genomics and Global Health is supported by the National Human Genome Research Institute, the National Institute of Diabetes and Digestive and Kidney Diseases, the Center for Information Technology and the Office of the Director at the National Institutes of Health (Z01HG200362).

\section{References}

1. Berg AH, Combs TP, Scherer PE. ACRP30/adiponectin: an adipokine regulating glucose and lipid metabolism. Trends Endocrinol Metab. 2002;13(2):84-89.

2. Berg AH, Combs TP, Du X, Brownlee M, Scherer PE. The adipocyte-secreted protein Acrp30 enhances hepatic insulin action. Nat Med. 2001;7(8):947-953.

3. Shetty S, Kusminski CM, Scherer PE. Adiponectin in health and disease: evaluation of adiponectin-targeted drug development strategies. Trends Pharmacol Sci. 2009;30(5):234-239.

4. Arita Y, Kihara S, Ouchi N, Takahashi M, Maeda K, Miyagawa J, Hotta K, et al. Paradoxical decrease of an adipose-specific protein, adiponectin, in obesity. Biochem Biophys Res Commun. 1999;257(1):79-83.

5. Ukkola O, Santaniemi M. Adiponectin: a link between excess adiposity and associated comorbidities? J Mol Med (Berl). 2002;80(11):696-702.

6. Fantuzzi G. Adipose tissue, adipokines, and inflammation. J Allergy Clin Immunol. 2005;115(5):911-919; quiz 920.

7. Juge-Aubry CE, Henrichot E, Meier CA. Adipose tissue: a regulator of inflammation. Best Pract Res Clin Endocrinol Metab. 2005;19(4):547-566.

8. Kern PA, Di Gregorio GB, Lu T, Rassouli N, Ranganathan G. Adiponectin expression from human adipose tissue: relation to obesity, insulin resistance, and tumor necrosis factor-alpha expression. Diabetes. 2003;52(7):1779-1785.

9. Mojiminiyi OA, Abdella NA, Al Arouj M, Ben Nakhi A. Adiponectin, insulin resistance and clinical expression of the metabolic syndrome in patients with Type 2 diabetes. Int J Obes (Lond). 2007;31(2):213-220.

10. Shikany JM, Lewis CE, Freedman BI, Arnett DK, Leiendecker-Foster C, Jones TL, Redden DT, et al. Plasma adiponectin concentrations and correlates in African Americans in the Hypertension Genetic Epidemiology Network (HyperGEN) study. Metabolism. 2007;56(8):1011-1016.

11. Dandona P, Aljada A, Chaudhuri A, Mohanty P, Garg R. Metabolic syndrome: a comprehensive perspective based on interactions between obesity, diabetes, and inflammation. Circulation. 2005;111(11):1448-1454.

12. Hanley AJ, Connelly PW, Harris SB, Zinman B. Adiponectin in a native Canadian population experiencing rapid epidemiological transition. Diabetes Care. 2003;26(12):3219-3225.

13. Yoon SJ, Lee HS, Lee SW, Yun JE, Kim SY, Cho ER, Lee SJ, et al. The association between adiponectin and diabetes in the Korean population. Metabolism. 2008;57(6):853-857.

14. Araneta MR, Barrett-Connor E. Adiponectin and ghrelin levels and body size in normoglycemic Filipino, African-American, and white women. Obesity (Silver Spring). 2007;15(10):2454-2462.

15. Considine RV, Premkumar A, Reynolds JC, Sebring NG, 
Ricks M, Sumner AE. Adiponectin and leptin in African Americans. Obesity (Silver Spring). 2008;16(2):428434.

16. Hanley AJ, Bowden D, Wagenknecht LE, Balasubramanyam A, Langfeld C, Saad MF, Rotter JI, et al. Associations of adiponectin with body fat distribution and insulin sensitivity in nondiabetic Hispanics and AfricanAmericans. J Clin Endocrinol Metab. 2007;92(7):26652671.

17. Doumatey AP, Lashley KS, Huang H, Zhou J, Chen G, Amoah A, Agyenim-Boateng K, et al. Relationships among obesity, inflammation, and insulin resistance in African Americans and West Africans. Obesity (Silver Spring). 2010;18(3):598-603.

18. Cohen SS, Gammon MD, Signorello LB, North KE, Lange EM, Fowke JH, Hargreaves MK, et al. Serum adiponectin in relation to body mass index and other correlates in black and white women. Ann Epidemiol. 2011;21(2):86-94.

19. Cohen SS, Gammon MD, North KE, Millikan RC, Lange EM, Williams SM, Zheng W, et al. ADIPOQ, ADIPOR1, and ADIPOR2 polymorphisms in relation to serum adiponectin levels and BMI in black and white women. Obesity (Silver Spring). 2011;19(10):20532062.

20. Beebe-Dimmer JL, Zuhlke KA, Ray AM, Lange EM, Cooney KA. Genetic variation in adiponectin (ADIPOQ) and the type 1 receptor (ADIPOR1), obesity and prostate cancer in African Americans. Prostate Cancer Prostatic Dis. 2010;13(4):362-368.

21. DeLoach S, Huan Y, Keith SW, Martinez Cantarin MP, Falkner B. Relationship of blood pressure and obesity with inflammatory cytokines among African Americans. Ther Adv Cardiovasc Dis. 2011;5(3):149-157.

22. Wassel CL, Pankow JS, Rasmussen-Torvik LJ, Li N, Taylor KD, Guo X, Goodarzi MO, et al. Associations of SNPs in ADIPOQ and subclinical cardiovascular disease in the multi-ethnic study of atherosclerosis (MESA). Obesity (Silver Spring). 2011;19(4):840-847.

23. Hicks C, Zhu X, Luke A, Kan D, Adeyemo A, Wu X, Cooper RS. A genome-wide scan of loci linked to serum adiponectin in two populations of African descent. Obesity (Silver Spring). 2007;15(5):1207-1214.

24. Aguilar-Salinas CA, Garcia EG, Robles L, Riano D, Ruiz-Gomez DG, Garcia-Ulloa AC, Melgarejo MA, et al. High adiponectin concentrations are associated with the metabolically healthy obese phenotype. J Clin Endocrinol Metab. 2008;93(10):4075-4079.

25. Morrison JA, Glueck CJ, Daniels S, Wang P, Horn P, Stroop D. Paradoxically high adiponectin and the healthy obese phenotype in obese black and white 16-year-old girls. Transl Res. 2010;156(5):302-308.

26. Karelis AD, Rabasa-Lhoret R. Inclusion of C-reactive protein in the identification of metabolically healthy but obese (MHO) individuals. Diabetes Metab. 2008;34(2):183-184.

27. Primeau V, Coderre L, Karelis AD, Brochu M, Lavoie ME, Messier V, Sladek R, et al. Characterizing the profile of obese patients who are metabolically healthy. Int J Obes (Lond). 2011;35(7):971-981.

28. Morrison JA, Glueck CJ, Daniels S, Wang P, Stroop D. Paradoxically high adiponectin in obese 16-year-old girls protects against appearance of the metabolic syndrome and its components seven years later. J Pediatr. 2011;158(2):208-214 e201.

29. Adeyemo A, Gerry N, Chen G, Herbert A, Doumatey A, Huang $\mathrm{H}$, Zhou J, et al. A genome-wide association study of hypertension and blood pressure in African Americans. PLoS Genet. 2009;5(7):e1000564.

30. Luke A, Durazo-Arvizu R, Rotimi C, Prewitt TE, Forrester T, Wilks R, Ogunbiyi OJ, et al. Relation between body mass index and body fat in black population samples from Nigeria, Jamaica, and the United States. Am J Epidemiol. 1997;145(7):620-628.

31. Matthews DR, Hosker JP, Rudenski AS, Naylor BA, Treacher DF, Turner RC. Homeostasis model assessment: insulin resistance and beta-cell function from fasting plasma glucose and insulin concentrations in man. Diabetologia. 1985;28(7):412-419.

32. Meilleur KG, Doumatey A, Huang H, Charles B, Chen $\mathrm{G}$, Zhou J, Shriner D, et al. Circulating adiponectin is associated with obesity and serum lipids in West Africans. J Clin Endocrinol Metab. 2010;95(7):3517-3521.

33. Lebovitz HE. The relationship of obesity to the metabolic syndrome. Int J Clin Pract Suppl. 2003134):1827.

34. Sumner AE, Zhou J, Doumatey A, Imoisili OE, Amoah A, Acheampong J, Oli J, et al. Low HDL-Cholesterol with Normal Triglyceride Levels is the Most Common Lipid Pattern in West Africans and African Americans with Metabolic Syndrome: Implications for Cardiovascular Disease Prevention. CVD Prev Control. 2010;5(3):75-80.

35. Wellen KE, Hotamisligil GS. Obesity-induced inflammatory changes in adipose tissue. J Clin Invest. 2003;112(12):1785-1788.

36. Karelis AD. Metabolically healthy but obese individuals. Lancet. 2008;372(9646):1281-1283.

37. Lara-Castro C, Doud EC, Tapia PC, Munoz AJ, Fernandez JR, Hunter GR, Gower BA, et al. Adiponectin multimers and metabolic syndrome traits: relative adiponectin resistance in African Americans. Obesity (Silver Spring). 2008;16(12):2616-2623.

38. Monzillo LU, Hamdy O, Horton ES, Ledbury S, Mullooly C, Jarema C, Porter S, et al. Effect of lifestyle modification on adipokine levels in obese subjects with insulin resistance. Obes Res. 2003;11(9):1048-1054.

39. Iwashima Y, Katsuya T, Ishikawa K, Kida I, Ohishi 
M, Horio T, Ouchi N, et al. Association of hypoadiponectinemia with smoking habit in men. Hypertension. 2005;45(6):1094-1100.

40. Koh KK, Quon MJ, Han SH, Ahn JY, Jin DK, Kim HS,
Kim DS, et al. Vascular and metabolic effects of combined therapy with ramipril and simvastatin in patients with type 2 diabetes. Hypertension. 2005;45(6):10881093. 